\title{
Études comparatives de plusieurs modèles de la rigidité des roulements sur le comportement dynamique d'une boîte de vitesses automobile
}

\author{
Adeline Bourdon a et CÉline Bordegaray \\ LaMCoS, INSA-Lyon, CNRS UMR5259, Domaine Scientifique de la Doua, Bâtiment Jean d'Alembert, 8 rue des Sciences, \\ 69621 Villeurbanne Cedex, France
}

Reçu le 18 octobre 2005, accepté le 9 février 2007

\begin{abstract}
Résumé - Les paliers à roulement sont présents dans de nombreux systèmes mécaniques et divers modèles numériques existent pour introduire leur comportement lors de simulations numériques représentatives du comportement dynamique du système mécanique considéré. L'objectif de cet article est dans un premier temps de présenter différentes modélisations existantes dans la littérature puis de comparer leur influence sur le comportement modal simulé d'une boîte de vitesses automobile.
\end{abstract}

Mots clés : Roulement / modélisation / simulation numérique / dynamique / mécanismes

Abstract - Comparative studies of several models of rolling bearing stiffness on the dynamic behaviour of an automotive gearbox. Rolling bearings are present in a large number of mechanical systems and various numerical models are used to introduce their behaviour in numerical simulations. The aim of this paper is first to make a literature review of the existing models of bearing and then to compare the influence of the numerical bearing model on the modal behaviour of an automotive gearbox.

Key words: Rolling bearing / modelling / numerical simulation / dynamics / mechanisms

\section{Introduction}

Les systèmes en rotation sont présents dans de larges domaines de l'industrie actuelle : production d'énergie, aéronautique, automobile ... La modélisation de leur comportement dynamique est l'objet de nombreux travaux de recherche dont le but est de comprendre les phénomènes vibratoires mis en jeux afin de mieux les contrôler et de développer des systèmes rotatifs plus résistants et plus silencieux. Beaucoup de ces travaux se focalisent sur la modélisation de la source de l'excitation : dentures pour les rotors engrenants, balourds et effets gyroscopiques pour les rotors «simples». La majorité de ces approches intègrent les arbres par l'intermédiaire de modèles à paramètres localisés [1-7] ou de modèles continus [8-12] mais les supports et les carters sont souvent considérés comme infiniment rigides et non pris en compte et les roulements supportant les arbres sont modélisés de manière beaucoup moins élaborée que le reste du système. Néanmoins, des travaux plus récents commencent à mettre en évidence l'influence non négligeable des

\footnotetext{
a Auteur correspondant : adeline.bourdon@insa-lyon.fr
}

roulements et donc de leur modélisation, sur le comportement dynamique simulé de ces systèmes tant par leur rôle de conditions aux limites dans les modèles « locaux» mais également comme élément de couplage entre les degrés de liberté et de transmission de la vibration aux carters $[8-11,13]$.

L'objectif de cet article est de répertorier les méthodes actuelles de modélisation de la rigidité des roulements et de les comparer. Pour cela, quatre modélisations seront retenues et intégrées dans un modèle dynamique d'un système à 3 arbres et à engrenages cylindriques. Les modes propres obtenus avec les différents modèles de roulements seront comparés.

\section{Les roulements dans la littérature}

Comme tous les organes mécaniques, les paliers à roulement influencent le comportement dynamique des systèmes mécaniques de différentes façons. Ils modifient la masse et donc l'inertie du système, contribuent à la flexibilité, sont source d'amortissement et éventuellement d'excitation. Un modèle idéal de roulement devrait 
intégrer ces différents aspects. Dans cet article, seule l'influence de la modélisation de leur flexibilité sur le comportement dynamique simulé sera traitée. En effet, l'estimation de la masse et des inerties d'un roulement ne présente a priori pas de grande difficulté, elles peuvent donc être introduites dans un modèle dynamique à l'aide d'éléments ponctuels. Les problèmes de modélisation de l'amortissement dû aux roulements, beaucoup plus complexes, rejoignent les problèmes génériques de la prise en compte de l'amortissement dans les modèles dynamiques et représentent à eux seuls matière à de nombreux travaux. La modélisation la plus courante est l'utilisation d'amortissements modaux. Néanmoins, certains auteurs évoquent la possibilité de modéliser le roulement par un système « ressort-amortisseur » sans préciser comment la valeur numérique de l'amortisseur équivalent est obtenue. Lim [14] évoque par exemple la possibilité de prendre en compte l'amortissement visqueux dû aux paliers à roulements par une matrice proportionnelle à la matrice de rigidité, M. Li [5] introduit une matrice d'amortissement $2 \times 2$ pleine. Dietl [15] propose une approche théorique et expérimentale pour calculer l'amortissement dans les roulements, cette approche est relativement complexe et difficile à introduire dans une modélisation plus générale. Enfin, les paliers à roulement constituent une source potentielle d'excitation due principalement [16] au désalignement des arbres, à l'état de surface des éléments roulants et des pistes de roulement, aux instabilités des cages, aux entrées/sorties des éléments roulants dans la zone chargée ... Ces excitations, de fréquence directement reliée à la vitesse de rotation des arbres par des paramètres géométriques invariants, sont généralement négligées devant celles introduites par l'engrènement ou les effets gyroscopiques et de balourds [16].

\subsection{Généralités sur la modélisation de la rigidité}

Dans le cadre des simulations numériques focalisées à l'engrènement $[2-7,17,18]$ ou lors de la modélisation de rotors « simples » $[10,19-23]$ les roulements représentent les conditions aux limites du modèle. Pour les études dynamiques plus complètes permettant de décrire la transmission des vibrations, les paliers sont les éléments de liaison entre le rotor et le carter. L'ensemble des modèles proposés dans la littérature est basé sur une linéarisation plus ou moins explicite de la relation entre les efforts appliqués aux roulements et les déplacements relatifs des bagues. Les différentes modélisations dynamiques de la rigidité des paliers à roulement découlent de la manière dont cette relation est identifiée. Dans tous les cas, il s'agira d'une rigidité $K_{\mathrm{t}}$ linéarisée au voisinage du chargement statique. Suivant les approches, cette grandeur est soit un scalaire soit une matrice qui permet d'écrire, autour d'un point de fonctionnement défini par une charge $Q$ et une déflection $\delta$ la relation :

$$
\Delta Q=K_{\mathrm{t}} \cdot \Delta \delta
$$

\subsection{Modélisation par des rigidités élémentaires}

La modélisation la plus classiquement utilisée $[1,4,8-10,19,24]$ consiste à introduire un ensemble de rigidités élémentaires susceptibles de représenter les raideurs axiales et radiales du roulement considéré. La manière dont les valeurs de ces rigidités sont obtenues est rarement explicitée dans les publications. Elles peuvent être obtenues de manière numérique en utilisant par exemple les formules de Palmgren [25] ou de Krämer [26] qui à partir des caractéristiques génériques des roulements (diamètres, nombre de corps roulant ...) et d'hypothèses simplificatrices (pas de jeux, environnement rigide, angles de contact ne variant pas ...) donnent des valeurs approchées de la relation efforts-déplacements. Ces formules correspondent à des cas de charges distincts (purement radiale ou purement axiale), ce qui implique un découplage au niveau des roulements entre les mouvements radiaux (flexion des arbres) et axiaux (extension). Synthétiquement, ces relations s'expriment par :

$$
\delta=\phi(F, D, \alpha, \ldots)
$$

où $\delta$ représente l'écrasement élastique, $F$ les efforts extérieurs appliqués au roulement, $D, \alpha \ldots$ des paramètres géométriques du roulement (diamètres, angle de contact ...).

Le détail des formules de Palmgren et Kramer est donné dans les annexes 1 et 2. La ré-écriture de ces formules sous la forme

$$
\Phi=\gamma(\delta, D, \alpha, \ldots)
$$

et le calcul de la dérivée partielle par rapport à $\delta$ au point d'équilibre statique permettent d'obtenir la valeur de la rigidité élémentaire à introduire dans le modèle numérique.

Ces rigidités élémentaires peuvent également être obtenues de manière expérimentale. E.R. Marsh [27] propose par exemple une méthode expérimentale basée sur les matrices d'impédance, il définit le nombre de mesures minimales à réaliser et étudie l'influence de la position des capteurs. Néanmoins, ces modèles simplifiés ne permettent pas de prendre en compte de manière satisfaisante les mécanismes de transfert vibratoire entre l'excitation interne au système et la réponse des carters [14, 28-30]. Pour les rotors engrenants, T.C. Lim [14] signale que les modèles « simples », n'utilisant que des raideurs purement radiales ne permettent pas de transmettre des moments et qu'ils sont donc inadéquats pour analyser la transmission dynamique des efforts au travers des roulements et les sollicitations des carters. C. Bard [31] en exploitant simultanément des modèles numériques et des données expérimentales a mis en évidence l'importance des rigidités des paliers à roulement qui conditionnent pour une grande part la localisation des vitesses critiques. Il conclut qu'un recalage correct de l'ensemble des vitesses critiques pour les dentures, passe obligatoirement par une modélisation complète des paliers et la prise en compte de l'ensemble des degrés de liberté. Pour cela, 
des modélisations quasi-statiques préalables permettant de prendre en compte aussi bien la géométrie locale que le positionnement global des éléments sont nécessaires.

\subsection{Modélisation par des matrices $5 \times 5$}

Depuis les années 1980, plusieurs auteurs ont proposé d'améliorer les modèles précédents en introduisant des couplages entre tous les mouvements relatifs des bagues. Le principe de ces approches est de réaliser un modèle quasi-statique du roulement isolé de son environnement, de lui appliquer un torseur d'efforts représentatif des efforts de fonctionnement vus par le roulement et d'en déduire une matrice de rigidité linéarisée, c'est cette matrice qui est ensuite introduite dans des modèles dynamiques. La section suivante présente un historique de la réalisation de ces modèles.

En 1982, M.D. Rajab [32] propose d'introduire les couplages entre le déplacement radial et la rotation selon le plan de flexion de la bague intérieure par rapport à la bague extérieure. Mais T.C. Lim [14] signale que l'analyse de M.D. Rajab était erronée et que la matrice de raideur résultante était incorrecte, de même pour les résultats de W.B. Young [33] qui avait étendu les résultats de M.D. Rajab à une matrice de dimension 3. Y. Kang [34] évoque une matrice à 8 coefficients intégrant les termes de couplages mais en pratique dans la publication évoquée seuls les termes radiaux sont introduits. La tendance actuelle s'oriente vers une définition de modèles généraux matriciels $[14,28,35,36]$ permettant de prendre en compte les couplages entre les déplacements généralisés relatifs des bagues l'une par rapport à l'autre. Ces modèles s'appuient sur une approche quasi-statique afin de calculer l'équilibre des roulements et la répartition des charges par une approche systématique avec une prise en compte plus fine de la géométrie interne des roulements : introduction des jeux, des courbures et bombés des bagues. S. Andreason [37] propose des modèles matriciels analytiques permettant de déterminer, à partir des déplacements relatifs des centres de courbure des bagues, la répartition des charges, les angles de contact et l'équilibre des corps roulants pour les roulements à billes à gorge profonde. Pour les rouleaux coniques, ses modèles prennent en compte les interactions entre le rouleau, les pistes et l'épaulement. Pour les roulements à rouleaux cylindriques, T.A. Harris [38, 39] décrit une méthode de calcul de la charge le long de la génératrice du rouleau qui tient compte du bombé. J.Y. Liu $[40,41]$ a affiné les modèles de S. Andreason et de T.A. Harris pour les roulements à rouleaux en introduisant les effets des accélérations centrifuges et le moment gyroscopique de chaque corps roulant. Le modèle proposé par J.M. de Mul [35] est basé sur une approche matricielle et une description vectorielle de la géométrie. Pour les roulements à billes, il permet de calculer la répartition des charges, les positions et les angles de contact. Pour les roulements à rouleaux, il prend en compte les interactions entre les rouleaux et les épaulements. S'appuyant sur les travaux précédents, Lim [14,28] propose une approche matricielle pouvant s'intégrer dans un modèle éléments-finis. Dans ses modèles, les bagues sont considérées comme indéformables. Les bagues extérieures sont encastrées et les efforts sont appliqués sur les bagues intérieures ramenées en un noud de l'axe. Ces nœuds peuvent être distincts des « centres de rotation» des roulements, et des moments de flexion peuvent être appliqués. Les roulements sont alors introduits dans les modèles dynamiques par l'intermédiaire de matrices de rigidité $(5 \times 5)$ couplant les 5 degrés de liberté du noud de la bague intérieure, la rotation suivant l'axe du roulement étant évidemment libre. Plus récemment, X. Hernot [42] propose une méthode analytique pour calculer la matrice de rigidité associée aux roulements à billes à contact oblique permettant d'introduire des précharges.

Pour des mécanismes de transmission de puissance tels les boîtes d'hélicoptères ou les boîtes de vitesses automobiles, des études ont montré [30, 36, 38, 39, 43-45] que l'augmentation de la souplesse des arbres et des carters, couplée à la forte non-linéarité des paliers à roulement, interdit d'isoler le palier et le reste de la structure comme réalisé dans les modèles de la section précédente. L'influence de la flexibilité de l'environnement ne peut pas être négligée, et les bagues des roulements ne peuvent pas toujours être considérées comme indéformables. Des études réalisées par A.B. Jones [43] ont montré que dans certaines conditions, la durée de vie des roulements déterminée avec la prise en compte de la déformabilité des bagues, était inférieure à celle obtenue avec l'hypothèse de bagues indéformables.

\subsection{Modélisation prenant en compte la flexibilité statique de l'environnement}

S'appuyant sur les travaux précédents, A. Bourdon $[30,36]$ a mis en place un principe de modélisation « unifiée » des roulements à billes, à rouleaux cylindriques et à rouleaux coniques, pouvant s'introduire dans une modélisation éléments-finis classique, en vue d'une résolution d'un problème non-linéaire. Ces éléments prennent en compte la géométrie interne des paliers à roulement et permettent d'obtenir les efforts exercés par les bagues et les épaulements sur le corps roulant, les angles de contact ou de désalignement, la matrice de rigidité linéarisée associée à chaque corps roulant.

L'idée essentielle de ces travaux est de discrétiser le roulement en corps roulants (billes ou rouleaux), et d'associer à chacun d'eux un élément de liaison numérique. L'approche par la méthode des forces, classique chez les roulementiers $[28,46]$ a été abandonnée au profit de la méthode des déplacements afin de mettre en place une approche globale permettant de faire le lien entre les calculs de roulements et le calcul de structure. Cet élément corps roulant relie un nœud de la bague intérieure à un nœud de la bague extérieure par l'intermédiaire d'une matrice de rigidité de dimension $10 \times 10$. Cette matrice établit le couplage entre les 10 degrés de liberté (ddl) actifs $(2 \times 3$ ddl de translation et $2 \times 2$ ddl de rotation) et 

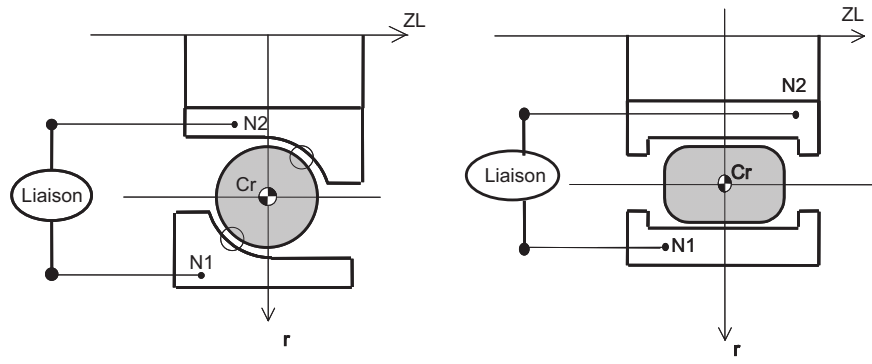

Fig. 1. Discrétisation des bagues pour les roulements à billes et à rouleaux.

assure la transmission de 3 forces et de 2 moments. Les degrés de liberté correspondant à la rotation du roulement sont, évidemment, libres de tout couplage et aucun moment n'est transmis suivant cette direction. Pour cela, les bagues intérieures et extérieures sont discrétisées, et la discrétisation doit vérifier les hypothèses suivantes:

- les sections transversales des bagues sont indéformables, ce qui revient à assimiler les bagues à des poutres courbes,

- à chaque corps roulant correspond un nœud de la bague extérieure et un noud de la bague intérieure. Ces nœuds sont situés dans le plan radial contenant le centre du corps roulant comme schématisé sur la figure 1 .

Il s'agit alors de déterminer pour chaque corps roulant, supposé indéformable sauf ponctuellement au niveau des contacts, et pour des déplacements donnés des nœuds d'attache $\mathrm{N}_{1}$ et $\mathrm{N}_{2}$, les déplacements élémentaires du centre $\mathrm{Cr}$ du corps roulant assurant l'équilibre de ce dernier dans le plan radial (équilibre des forces et des moments). En effet, pour des déplacements relatifs des bagues connus, le corps roulant va être soumis à des efforts de contact au niveau des pistes de roulement et des épaulements. Lorsqu'ils s'appliquent, ces efforts sont évalués par la théorie de Hertz (cas par exemple des roulements à billes). Ainsi

$$
Q=C_{\mathrm{f}} \cdot(\delta)^{n}
$$

où $Q$ représente l'effort de contact, $\delta$ représente le rapprochement des corps, $n=3 / 2$ et la constante $C_{\mathrm{f}}$ est déduite de la géométrie et des matériaux des solides en contact. Lorsque la théorie de Hertz n'est plus valide (cas par exemple des roulements à rouleaux) ces efforts sont évalués à partir d'une approche simplifiée discrétisant les lignes de contact en tranches minces indépendantes les unes des autres et pour lesquelles la relation effortdéplacement est choisie sous une forme similaire à celle décrite par l'équation (4) avec $n=10 / 9$. Le principe de calcul pour les roulements à rouleaux est largement inspiré des travaux de T.A. Harris [38,39].

La position d'équilibre du système bagues-corps roulant est calculée par une boucle itérative de NewtonRaphson (Fig. 2). À chaque itération sont déterminés les efforts exercés sur le corps roulant par chaque bague et ses éventuels épaulements. Ce calcul se fait en tenant compte des jeux ou des précharges assimilées à des jeux négatifs et de la géométrie précise du roulement (rayons de courbure, de dépouille, bombés ...).

Lorsque l'équilibre est atteint (vecteur des efforts résiduels égal au vecteur nul), les efforts entre le corps roulant, les bagues et les épaulements sont connus, ainsi que les matrices de rigidité tangente entre le corps roulant et les différentes bagues. Une matrice de rigidité élémentaire linéarisée peut être déterminée entre les nœuds de la bague intérieure et extérieure et associée à l'élément « corps roulant ». La méthodologie détaillée du calcul de l'équilibre des corps roulants est décrite dans la publication précédente ainsi que dans l'annexe B1 de la thèse associée [30,36].

L'avantage de cette approche est de permettre de traiter aussi bien le cas des paliers montés dans un environnement rigide avec des bagues indéformables, que celui des roulements montés dans un environnement flexible avec des bagues déformables. Les différences essentielles entre les éléments proposés ici et l'approche de J.M. de Mul [35] sont que les basculements des bagues dans le plan radial sont pris en compte et qu'une matrice de rigidité linéarisée $(10 \times 10)$ est définie pour chaque corps roulant. L'introduction dans les modèles dynamiques de ces matrices de rigidité linéarisée associée se traduit par l'introduction pour chaque corps roulant d'une matrice de rigidité pleine couplant 5 degrés de liberté d'un nœud de la bague intérieure à 5 degrés de liberté d'un nœud de la bague extérieure. Cette approche permet d'introduire facilement les effets dynamiques du carter [30,47-49]. Des études, non présentées dans cet article [50] ont montré que les positions relatives des corps roulants par rapport aux bagues de roulement n'influençaient quasiment pas les termes des matrices de rigidité obtenues.

\section{Modélisation dynamique d'un système multi-arbres sur roulements}

\subsection{Description du système considéré et modélisation}

Le système considéré est un réducteur à trois étages composé d'engrenages hélicoïdaux, issu d'une application réelle (Fig. 3). L'arbre d'entrée est supporté par deux roulements coniques, l'arbre intermédiaire par un roulement à billes à gorge profonde et un roulement à rouleaux cylindriques, l'arbre de sortie par deux roulements coniques. Cinq engrenages hélicoïdaux sont présents entre l'arbre d'entrée et l'arbre intermédiaire, ce qui permet d'envisager pour un couple d'entrée fixé des torseurs d'efforts différents sur les roulements. Le détail de la géométrie de ce système est donné dans les tableaux 1 à 3 .

Si on excepte les roulements dont la description de la modélisation fera l'objet d'un paragraphe spécifique, la modélisation du système est classique et très proche de celle de M. Kubur [8]. Les arbres sont modélisés par des éléments-finis de poutre à 2 nœuds et 6 ddl par nœud. On considère un fonctionnement sous charge, sans perte de contact dans les engrenages. Ces derniers sont assimilés 


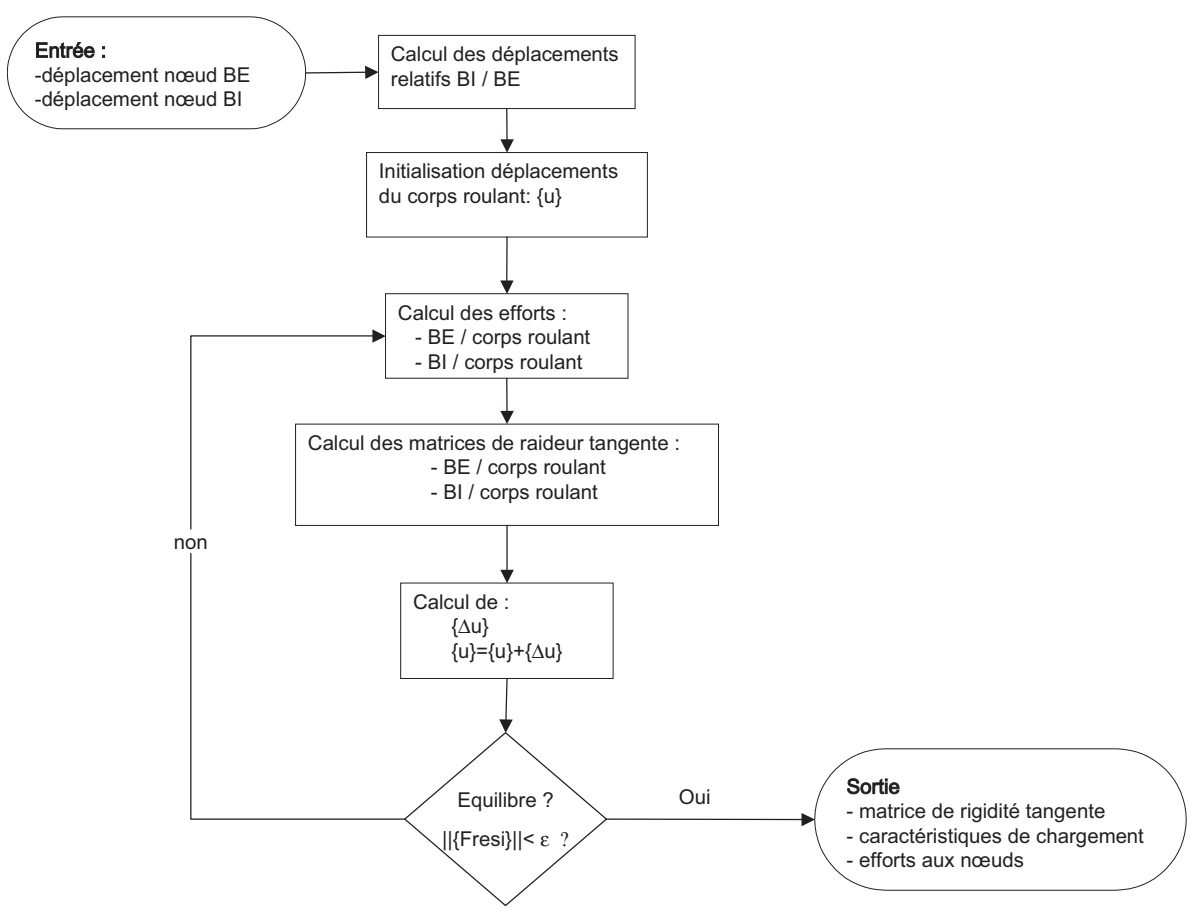

Fig. 2. Processus de calcul de l'équilibre des corps roulants.

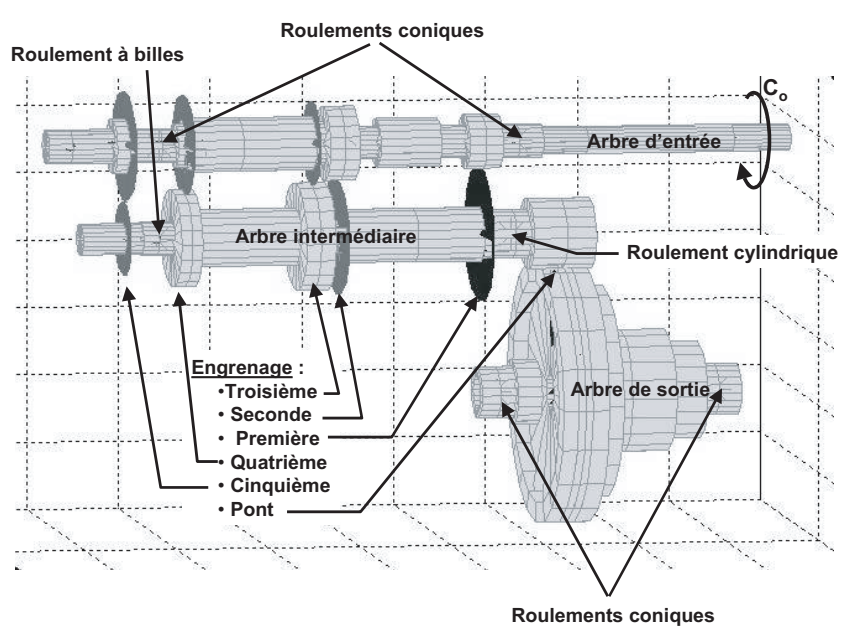

Fig. 3. Réducteur considéré.

à des corps rigides connectés par une rigidité moyenne $k_{\mathrm{g}}$ située dans le plan d'action dans la direction normale à la surface de denture et définie par les angles de pression $\alpha$ et d'hélice $\beta$. De très nombreux travaux décrivent largement ce principe de modélisation qui ne sera pas plus détaillé. Pour les engrenages en prise, la rigidité moyenne d'engrènement a été fixée à $3 \times 10^{8} \mathrm{~N} . \mathrm{m}^{-1}$. Cette valeur correspond à la rigidité moyenne de l'engrenage de $4^{\mathrm{e}}$ (sans correction de denture) soumis à un couple $C_{0}$. Des études préalables ont montré la faible influence de cette valeur sur les modes [30] propres du système tant du point de vue des fréquences que des formes propres. Pour l'engrenage de $1^{\text {re }}$ par exemple considérer une rigidité d'engrènement de $1 \times 10^{8} \mathrm{~N} . \mathrm{m}^{-1}$ ou de $1 \times 10^{13}{\mathrm{~N} . \mathrm{m}^{-1}}^{-1}$ entraîne des modifications inférieures
Tableau 1. Caractéristiques des matériaux des arbres.

\begin{tabular}{ccc}
\hline$E(\mathrm{~Pa})$ & $G(\mathrm{~Pa})$ & $\rho\left(\mathrm{kg} \cdot \mathrm{m}^{-3}\right)$ \\
\hline $2,1 \mathrm{E} 11$ & $8,14 \mathrm{E} 10$ & $7,8 \mathrm{E} 3$ \\
\hline
\end{tabular}

à $7 \%$ des fréquences propres. Pour les autres engrenages présents dans le modèle mais possédant un pignon fou, la rigidité moyenne est nulle ce qui empêche toute transmission d'effort. Les masses et inerties des pignons, des roulements et également des dispositifs de synchronisation sont introduites sous la forme d'éléments ponctuels. Le modèle dynamique final est formé de 50 éléments de poutre soit 53 nœuds et 318 degrés de liberté.

\subsection{Modélisation des roulements}

Comme vu dans les sections précédentes, de nombreuses approches existent dans la littérature pour modéliser le comportement dynamique des roulements. Il apparaît donc nécessaire de comparer ces modèles afin d'estimer les conséquences du choix de l'un ou de l'autre sur les résultats des simulations. Quatre modèles dynamiques de roulement notés a, b, c et d sont envisagés. Pour chacun d'eux, les rigidités linéarisées (scalaires ou matricielles) sont déterminées à l'aide d'un modèle statique du roulement pour un chargement équivalent à un couple $C_{0}=215 \mathrm{~N} \mathrm{~m}^{-1}$ appliqué en entrée de la boîte de vitesses. Les différences entre les 4 modèles présentés ci-après proviennent de la modélisation statique préalable utilisée.

Le modèle a est constitué de rigidités scalaires calculées par les formules de Palmgren [25], les efforts 
Tableau 2. Dimensions des arbres.

\begin{tabular}{cccccccccc}
\hline & \multicolumn{3}{c}{ Arbre d'entrée } & \multicolumn{3}{c}{ Arbre intermédiaire } & \multicolumn{3}{c}{ Arbre de sortie } \\
\hline Segment & $\begin{array}{c}\text { Diamètre } \\
\text { extérieur }(\mathrm{mm})\end{array}$ & $\begin{array}{c}\text { Diamètre } \\
\text { intérieur }(\mathrm{mm})\end{array}$ & $\begin{array}{c}L \\
(\mathrm{~mm})\end{array}$ & $\begin{array}{c}\text { Diamètre } \\
\text { extérieur } \\
(\mathrm{mm})\end{array}$ & $\begin{array}{c}\text { Diamètre } \\
\text { intérieur } \\
(\mathrm{mm})\end{array}$ & $\begin{array}{c}L \\
(\mathrm{~mm}) \\
(\mathrm{mm})\end{array}$ & $\begin{array}{c}\text { Diamètre } \\
\text { extérieur } \\
(\mathrm{mm})\end{array}$ & $\begin{array}{c}\text { Diamètre } \\
\text { intérieur }\end{array}$ & $\begin{array}{c}L \\
(\mathrm{~mm})\end{array}$ \\
\hline 1 & 20 & 0 & 135 & 52 & 7,8 & 34 & 38 & 26 & 21,45 \\
2 & 25 & 0 & 32,3 & 36 & 7,8 & 48 & 72 & 56 & 18 \\
3 & 40,8 & 0 & 9 & 25 & 7,8 & 62 & 90 & 77 & 34 \\
4 & 24 & 0 & 15 & 80 & 7,8 & 16 & 156 & 77 & 11 \\
5 & 36 & 0 & 33 & 42 & 7,8 & 56 & 194 & 164 & 9 \\
6 & 24 & 0 & 13 & 72 & 7,8 & 11,45 & 194 & 26 & 8 \\
7 & 5 & 0 & 16 & 25 & 7,8 & 17 & 194 & 164 & 8 \\
8 & 32 & 0 & 81 & 23 & 7,8 & 33 & 48 & 26 & 113 \\
9 & 25 & 0 & 70 & & & & 38 & 26 & 19 \\
\hline
\end{tabular}

Tableau 3. Paramétre des engrenages.

\begin{tabular}{ccccc}
\hline $\begin{array}{c}\text { Rapport de } \\
\text { vitesses }\end{array}$ & $\begin{array}{c}\text { Rayon base } \\
\text { Rb1 (mm) }\end{array}$ & $\begin{array}{c}\text { Rayon base } \\
\text { Rb2 }(\mathrm{mm})\end{array}$ & $\begin{array}{c}\text { Angle de pression } \\
(\mathrm{deg})\end{array}$ & $\begin{array}{c}\text { Angle d'hélice } \\
(\mathrm{deg})\end{array}$ \\
\hline 1 & 14,35 & 49,6 & 24,018 & 29,3 \\
2 & 22,92 & 42,86 & 20 & 27,3 \\
3 & 30,78 & 35,34 & 19,11 & 26,8 \\
4 & 36,76 & 30,23 & 16,85 & 26,6 \\
5 & 40,35 & 26,61 & 16,90 & 27,74 \\
6 (pont) & 21,90 & 88,97 & 21,29 & 19,71 \\
\hline
\end{tabular}

radiaux et axiaux appliqués à chaque roulement sont déterminés en calculant l'équilibre des arbres soumis aux efforts des engrenages en assimilant les roulements à des appuis simples. Ce modèle permet donc de prendre en compte le chargement global des roulements mais suppose l'absence de moment.

Le modèle $\mathbf{b}$ est une matrice $5 \times 5$ correspondant au modèle proposé par T.C. Lim [14,28]. Il permet donc d'introduire les effets de la répartition des charges pour des bagues et un environnement supposés indéformables. Le chargement est introduit par le torseur des efforts calculé aux nœuds de modélisation de la bague intérieure. Les couplages éventuels entre roulements (contact oblique, rouleaux coniques) dus aux efforts induits doivent être calculés et introduits explicitement.

Le modèle c correspond à une discrétisation en corps roulant comme présentée à la section 2.4 appliquée au système arbres-engrenages dans un carter rigide. Cette approche permet de prendre en compte implicitement les couplages entre roulements et éventuellement des précharges. Le chargement est appliqué sous la forme d'un couple $C_{0}$ en entrée du système.

Le modèle d enfin est comparable au modèle c mais le modèle statique utilisé prend en compte la flexibilité statique du carter et la déformabilité des bagues extérieures. C'est le modèle le plus complet, les résultats obtenus avec ce modèle seront par la suite pris comme référence. La rigidité du carter est introduite à l'aide d'une matrice de rigidité condensée obtenue à partir d'un maillage volumique du carter (37000 nouds, 20000 éléments). Un descriptif plus détaillé de cette modélisation est donné dans la publication [36]. Néanmoins, les engrenages étant modélisés par des rigidités linéaires (cf. Sect. 3.1), cette modélisation ne permet pas le calcul simultané des efforts transmis aux paliers et de l'erreur statique de transmission.

Il est intéressant de noter que pour les modèles c et $\mathrm{d}$, à l'issue des calculs statiques, une matrice de rigidité tangente $10 \times 10$ est obtenue pour chacun des $Z$ corps roulants. Après assemblage dans le modèle dynamique de ces $Z$ matrices et élimination des lignes et des colonnes associées aux ddl bloqués (bague extérieure), une matrice de rigidité tangente $(5 \times 5)$ est obtenue comme pour le modèle b. L'influence de la modélisation sur ces matrices de rigidités a été présentée [36].

\subsection{Les outils d'analyse}

Afin de pouvoir comparer les modes propres obtenus pour les différentes modélisations des roulements, deux outils d'analyse sont proposés : les matrices de corrélation modale et les analyses énergétiques.

La matrice de corrélation modale aussi appelée matrice de MAC (Modal Assurance Criterion), est utilisée pour comparer deux ensembles de modes propres $\left[Y^{1}\right]$ et $\left[Y^{2}\right]$, en particulier pour le recalage calcul-essais.

Soit :

$$
\begin{aligned}
& {\left[Y^{1}\right]=\left[\left\{y_{1}^{1}\right\} \ldots\left\{y_{k}^{1}\right\} \ldots\left\{y_{N_{1}}^{1}\right\}\right]} \\
& {\left[Y^{2}\right]=\left[\left\{y_{1}^{2}\right\} \ldots\left\{y_{k}^{2}\right\} \ldots\left\{y_{N_{2}}^{2}\right\}\right]}
\end{aligned}
$$

deux séries de modes propres. Le nombre de modes déterminés dans chaque ensemble peut être différent 
$(N 1 \neq N 2)$ mais les ddl modélisés doivent être identiques. La matrice de MAC associée à ces deux séries de vecteurs est définie par :

$$
\underset{\substack{i=1 \text { à } N_{1} \\ j=1 \text { à } N_{2}}}{(\mathrm{MAC})_{i, j}}=\frac{\left|T\left\{y_{i}^{1}\right\} \cdot\left\{y_{j}^{2}\right\}\right|^{2}}{\left\|\left\{y_{i}^{1}\right\}\right\|^{2}\left\|\left\{y_{j}^{2}\right\}\right\|^{2}}
$$

Cette matrice permet de quantifier le degré de corrélation entre deux vecteurs par un seul nombre compris entre 0 et $1(\mathrm{MAC})_{i, j}=1$ indique que le $i^{\text {ème }}$ vecteur de la première série est colinéaire au $j$ ème vecteur de la seconde série, c'est-à-dire que les déformées propres sont identiques, il y a une corrélation parfaite. En revanche, $(\mathrm{MAC})_{i, j}=0$ indique que les deux vecteurs sont orthogonaux, les déformées propres sont très différentes ou dans des plans différents. Dans la pratique, une valeur supérieure à 0,8 indique en général une bonne corrélation.

L'analyse énergétique [49,50] consiste à décomposer les énergies de la structure en énergies élémentaires de flexion, de torsion et d'extension. Cette décomposition permet de visualiser le couplage des effets, et ultérieurement, de choisir des modes propres représentatifs du système à étudier.

Pour un mode conservatif de pulsation propre $\omega_{i}$ et de vecteur propre $\phi_{i}$, il est sans intérêt de calculer l'énergie de déformation globale $W^{\mathrm{g}}$ ou l'énergie cinétique globale $T^{\mathrm{g}}$ qui sont toutes les deux égales à $1 / 2 \omega_{i}^{2}$, à cause des relations d'orthonormalité choisies pour les modes propres normés à 1 avec la matrice de masse :

$$
\begin{aligned}
W^{\mathrm{g}} & =\frac{1}{2}^{T}\left\{\phi_{i}\right\}\left[K_{\mathrm{g}}\right] \cdot\left\{\phi_{i}\right\}=\frac{1}{2} \omega_{i}^{2} \\
T^{\mathrm{g}} & =\frac{1}{2} \omega^{2^{T}}\left\{\phi_{i}\right\}\left[K_{\mathrm{g}}\right] \cdot\left\{\phi_{i}\right\}=\frac{1}{2} \omega_{i}^{2}
\end{aligned}
$$

Il est en revanche beaucoup plus intéressant de calculer l'énergie globale du système comme la somme des énergies élémentaires de chaque élément ou macroélément du modèle :

$$
\begin{aligned}
{\left[K_{\mathrm{g}}\right] } & =\sum_{\mathrm{e}}\left[K_{\mathrm{g}}^{\mathrm{e}}\right] \Rightarrow W_{\mathrm{i}}^{\mathrm{g}}=\frac{1}{2}^{T}\left\{\phi_{i}\right\}=\sum_{\mathrm{e}}\left[K_{\mathrm{g}}^{\mathrm{e}}\right]\left\{\phi_{i}\right\} \\
& =\sum_{\mathrm{e}} \frac{1}{2}^{T}\left\{\phi_{i}\right\}\left[K_{\mathrm{g}}^{\mathrm{e}}\right]\left\{\phi_{i}\right\}=\sum_{\mathrm{e}} W_{i}^{\mathrm{e}} \\
{\left[M_{\mathrm{g}}\right] } & =\sum_{\mathrm{e}}\left[M_{\mathrm{g}}^{\mathrm{e}}\right] \Rightarrow T_{\mathrm{i}}^{\mathrm{g}}=\frac{1}{2}{\omega_{i}^{2}}^{T}\{\phi,\} \sum_{\mathrm{e}}\left[M_{\mathrm{g}}^{\mathrm{e}}\right] \cdot\left\{\phi_{i}\right\} \\
& =\sum_{\mathrm{e}} \frac{1}{2} \omega_{i}^{2^{T}}\left\{\phi_{i}\right\}=\left[M_{\mathrm{g}}^{\mathrm{e}}\right]\left\{\phi_{i}\right\}=\sum_{\mathrm{e}} T_{i}^{\mathrm{e}}
\end{aligned}
$$

Cette décomposition permet de déterminer la contribution de chaque élément technologique aux déformées modales. Afin de pouvoir comparer les décompositions énergétiques de plusieurs modes, les énergies élémentaires $T^{\mathrm{e}}$ et $W^{\mathrm{e}}$ sont adimensionnées en les divisant par l'énergie globale du mode correspondant soit $1 / 2 \omega_{i}^{2}$.
Cette analyse peut être affinée en décomposant les énergies élémentaires en énergie de torsion, flexion et extension. Pour chaque élément il est facile d'écrire dans sa base locale que :

$$
\begin{aligned}
& K_{\mathrm{l}}^{\mathrm{e}}=K_{\mathrm{T}, 1}^{\mathrm{e}}+K_{\mathrm{F}, 1}^{\mathrm{e}}+K_{\mathrm{E}, 1}^{\mathrm{e}}=\sum_{\mathrm{s}} K_{\mathrm{s}, 1}^{\mathrm{e}} \\
& M_{\mathrm{l}}^{\mathrm{e}}=M_{\mathrm{T}, 1}^{\mathrm{e}}+M_{\mathrm{F}, 1}^{\mathrm{e}}+M_{\mathrm{E}, 1}^{\mathrm{e}}=\sum_{\mathrm{s}} M_{\mathrm{s}, 1}^{\mathrm{e}}
\end{aligned}
$$

avec

- $T$ : torsion

- $F$ : flexion

- $E$ : extension.

La connaissance de ces matrices découplées exprimées dans les bases locales permet de calculer les matrices découplées dans la base globale. Les énergies globales peuvent s'écrire :

$$
\begin{aligned}
W^{\mathrm{g}} & =\sum_{\mathrm{e}} \sum_{\mathrm{s}} \frac{1}{2}^{\mathrm{T}}\left\{\phi_{i}\right\}\left[K_{\mathrm{s}, \mathrm{g}}^{\mathrm{e}}\right] \cdot\left\{\phi_{i}\right\}=\sum_{\mathrm{e}} \sum_{\mathrm{s}} W_{\mathrm{s}}^{\mathrm{e}}(\mathrm{a}) \\
& =\sum_{\mathrm{s}} \sum_{\mathrm{e}} \frac{1}{2}^{\mathrm{T}}\left\{\phi_{i}\right\}\left[K_{\mathrm{s}, \mathrm{g}}^{\mathrm{e}}\right]\left\{\phi_{i}\right\}=\sum_{\mathrm{s}} W_{\mathrm{s}}^{\mathrm{g}} \quad(\mathrm{b}) \\
T^{\mathrm{g}} & =\sum_{\mathrm{e}} \sum_{\mathrm{s}} \frac{1}{2} \omega_{i}^{2 \mathrm{~T}}\left\{\phi_{i}\right\}\left[M_{\mathrm{s}, \mathrm{g}}^{\mathrm{e}}\right] \cdot\left\{\phi_{i}\right\}=\sum_{\mathrm{e}} \sum_{\mathrm{s}} T_{\mathrm{s}}^{\mathrm{e}}(\mathrm{a}) \\
& =\sum_{\mathrm{s}} \sum_{\mathrm{e}} \frac{1}{2} \omega_{i}^{2^{\mathrm{T}}}\left\{\phi_{i}\right\}\left[M_{\mathrm{s}, \mathrm{g}}^{\mathrm{e}}\right] \cdot\left\{\phi_{i}\right\}=\sum_{\mathrm{s}} T_{\mathrm{s}}^{\mathrm{g}} \quad(\mathrm{b})
\end{aligned}
$$

Deux sortes d'études énergétiques peuvent alors être réalisées : pour un mode, en utilisant les équations (11a) et (12a), il est possible d'examiner la répartition élémentaire des énergies découplées; pour plusieurs modes, en utilisant les équations (11b) et (12b), il est possible d'examiner la répartition globale des énergies découplées.

Afin d'appliquer cette méthode à l'étude des modes propres d'un système multi-rotors, il est nécessaire de définir pour chaque type d'élément utilisé, les matrices élémentaires découplées exprimées dans les bases locales. Pour les éléments de poutre, cette décomposition est standard et est obtenue sans difficulté. Pour les autres éléments, cette décomposition est plus arbitraire et a été réalisée en analogie avec les systèmes masses-ressorts : pour les éléments de type masse et inertie ponctuelle, l'effet de masse est comptabilisé comme de l'extension et l'effet de l'inertie comme de la torsion; pour les éléments d'engrenage et de roulement les effets sont comptabilisés en extension.

\section{Influence de la modélisation des roulements sur les modes propres du système}

Les études présentées dans cette partie ont pour objet d'estimer l'influence de la modélisation des roulements sur les modes propres du système conservatif. Afin d'obtenir des résultats correspondants à différents niveaux de chargement des paliers les 5 rapports de vitesse possibles sont considérés pour un même couple d'entrée. Pour 


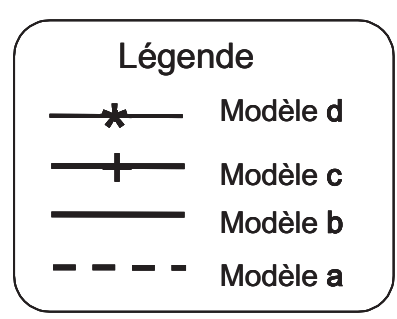

Troisième

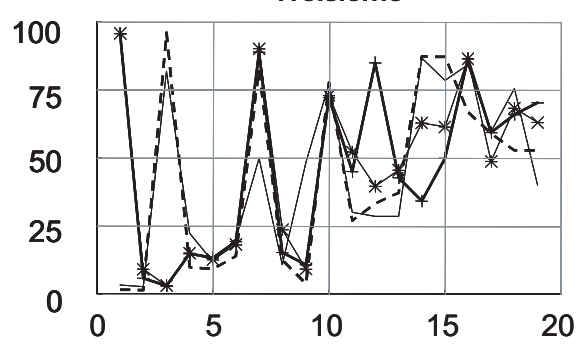

Première

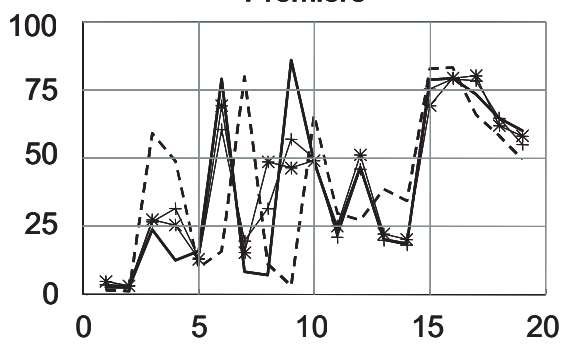

Quatrième

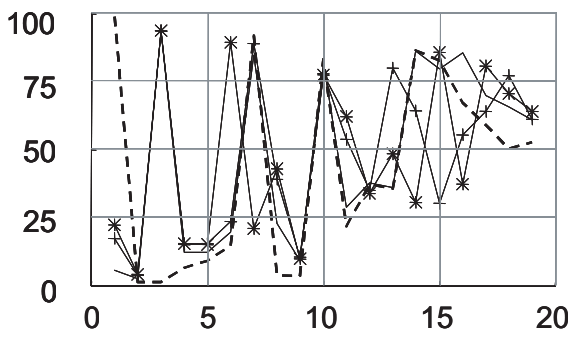

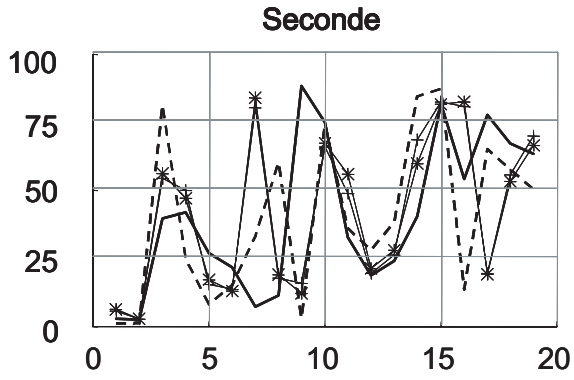

20

Fig. 4. Énergies potentielles dans les 6 roulements en fonction des modes.

Tableau 4. Bandes de fréquences correspondant aux 19 premiers modes propres (en Hertz).

\begin{tabular}{lccccc}
\hline & Première & Seconde & Troisième & Quatrième & Cinquième \\
\hline Modèle a & $196-2673$ & $196-2675$ & $196-2594$ & $153-2577$ & $196-2554$ \\
Modèle b & $195-2747$ & $194-2649$ & $194-2679$ & $190-2303$ & $191-2289$ \\
Modèle c & $192-2774$ & $189-2434$ & $166-2374$ & $178-2234$ & $184-2213$ \\
Modèle d & $191-2527$ & $188-2436$ & $164-2558$ & $173-2442$ & $179-2253$ \\
\hline
\end{tabular}

Tableau 5. Nombre de modes où plus de $50 \% / 75 \%$ d'énergie de déformation modale se situent dans les roulements.

\begin{tabular}{cccccc}
\hline \multicolumn{5}{c}{ Première Seconde Troisième Quatrième Cinquième } \\
\hline Modèle d & $7 / 2$ & $9 / 3$ & $10 / 3$ & $8 / 5$ & $7 / 3$ \\
Modèle c & $7 / 3$ & $9 / 3$ & $8 / 4$ & $10 / 5$ & $8 / 3$ \\
Modèle b & $7 / 4$ & $7 / 4$ & $8 / 6$ & $8 / 6$ & $8 / 5$ \\
Modèle a & $9 / 3$ & $7 / 3$ & $8 / 4$ & $8 / 5$ & $8 / 3$ \\
\hline
\end{tabular}

les 4 modèles de roulements et les 5 rapports de vitesse considérés les 19 premiers modes propres ont été calculés. Le tableau 4 regroupe les bandes de fréquences associées.

Bien que la modélisation de type d des roulements permette d'introduire les effets dynamiques du carter par l'intermédiaire de matrices de masse et de rigidité sousstructurées [30], les calculs de modes propres présentés ici ont été réalisés avec un carter rigide (les bagues extérieures des roulements sont encastrées) afin que les résultats obtenus avec les 4 types de modélisations des roulements puissent être comparés.

\subsection{Quantification de l'influence dynamique des roulements}

Pour les 19 premiers modes propres calculés et pour l'ensemble des modèles de roulements envisagés, les paliers apparaissent comme des organes contrôlant en moyenne plus de $40 \%$ des énergies de déformation modale. Ceci justifie a posteriori la nécessité d'évaluer l'influence de la modélisation de la rigidité des roulements. Le tableau 5 indique, pour chaque rapport de vitesse et chaque modèle de roulement, le nombre de modes où plus de, respectivement, $50 \%$ ou $75 \%$ des énergies de déformation sont localisés dans les roulements. Pour le modèle $\mathrm{d}$ en première il y a donc 7 modes où plus de $50 \%$ de l'énergie est localisée dans les roulements et 2 modes avec plus de $75 \%$ des énergies dans les roulements.

Quel que soit le rapport de vitesse considéré, chaque modèle possède entre 2 et 6 modes où plus de $75 \%$ des déformations ont lieu dans les roulements. L'analyse plus détaillée des résultats (Fig. 4) montre que ces modes sont d'autant plus énergétiques que le chargement statique est faible (rapport de vitesse élevé). Ils correspondent alors à des fréquences propres plus basses. Même en tenant compte des éventuels décalages de modes, les modèles a et $\mathrm{b}$ se distinguent des autres : les pics énergétiques ne correspondent pas à ceux des autres modèles ni en fréquence ni en amplitude. Le modèle a se particularise en première et seconde, par l'existence de modes énergétiques pour les roulements, dès les basses fréquences. La modélisation par des matrices de rigidité linéarisées couplant les 5 degrés de libertés et déterminées dans un environnement statique flexible (modèle $\mathrm{d}$ ), tend à augmenter l'influence des paliers sur le comportement modal de l'ensemble de la chaîne cinématique.

\subsection{Influence de la modélisation sur les modes propres}

Pour les différents rapports de vitesses, la colinéarité entre les vecteurs propres obtenus avec les modèles a, b, c et les 19 modes calculés du modèle de référence (modèle d) 
Tableau 6. Nombre de vecteurs propres pouvant être corrélé avec un MAC supérieur à 0,8 .

\begin{tabular}{lccccc}
\hline & Première & Seconde & Troisième & Quatrième & Cinquième \\
\hline Modèle a/d & 9 & 10 & 11 & 10 & 7 \\
Modèle b/d & 11 & 10 & 11 & 10 & 12 \\
Modèle c/d & 14 & 19 & 15 & 15 & 15 \\
\hline
\end{tabular}

a été évaluée à l'aide des matrices de MAC. Le tableau 6 indique le nombre de vecteurs corrélés à plus de $80 \%$ pour chaque configuration envisagée.

Les modèles a et $b$ apparaissent comme insuffisants pour décrire correctement les vecteurs propres du système puisque environ seulement $50 \%$ des modes peuvent être appariés en forme avec ceux du modèle d (Tab. 6). De plus des études détaillées non présentées ici montrent que le modèle a ne permet pas de déterminer correctement les premiers modes propres du système. En effet, pour l'ensemble des vitesses considérées, le modèle d possède 2 ou 3 modes de fréquences inférieures à $200 \mathrm{~Hz}$, qui sont plus ou moins représentés en fréquences dans le modèle a mais très mal en forme propre.

Pour les modes « appariables », les fréquences propres du modèle b sont supérieures à celles du modèle $d$. Par ailleurs, des modes corrélés en forme à plus de $80 \%$ entre ces 2 modèles peuvent être associés à des fréquences propres différentes. En revanche, d'autres modes voisins en fréquences ne sont pas corrélés en forme. Lors de la détermination des matrices de rigidité associées aux roulements le choix de l'environnement (rigide modèle b ou flexible modèle $d$ ) va donc modifier de manière découplée les fréquences propres et les vecteurs propres. Pour certains modes, la prise en compte d'un environnement flexible va modifier la forme ou l'orientation des vecteurs propres mais sans changer les fréquences propres. Inversement, pour d'autres modes, la prise en compte de l'environnement va se traduire par la diminution des fréquences propres mais sans modification sensible des vecteurs propres. D'autres modes enfin ne sont présents que dans un seul des deux modèles.

L'introduction de la flexibilité des arbres et des couplages entre les roulements à rouleaux coniques permet une bien meilleure représentation des vecteurs propres : $79 \%$ des vecteurs sont appariables entre le modèle c et le modèle $\mathrm{d}$ en forme et en fréquences. L'influence statique du carter apparaît pour des fréquences propres relativement « basses » et est surtout sensible lorsque les charges transmises sont élevées (première) où seule la moitié des modes de fréquence inférieure à $1000 \mathrm{~Hz}$ sont appariables en forme propre.

\subsection{Influence de la modélisation sur les densités modales}

Une analyse détaillée montre que les répartitions des fréquences propres ne sont pas identiques. La figure 5 donne dans le cas des rapports de première et de quatrième une illustration des densités modales pour chaque

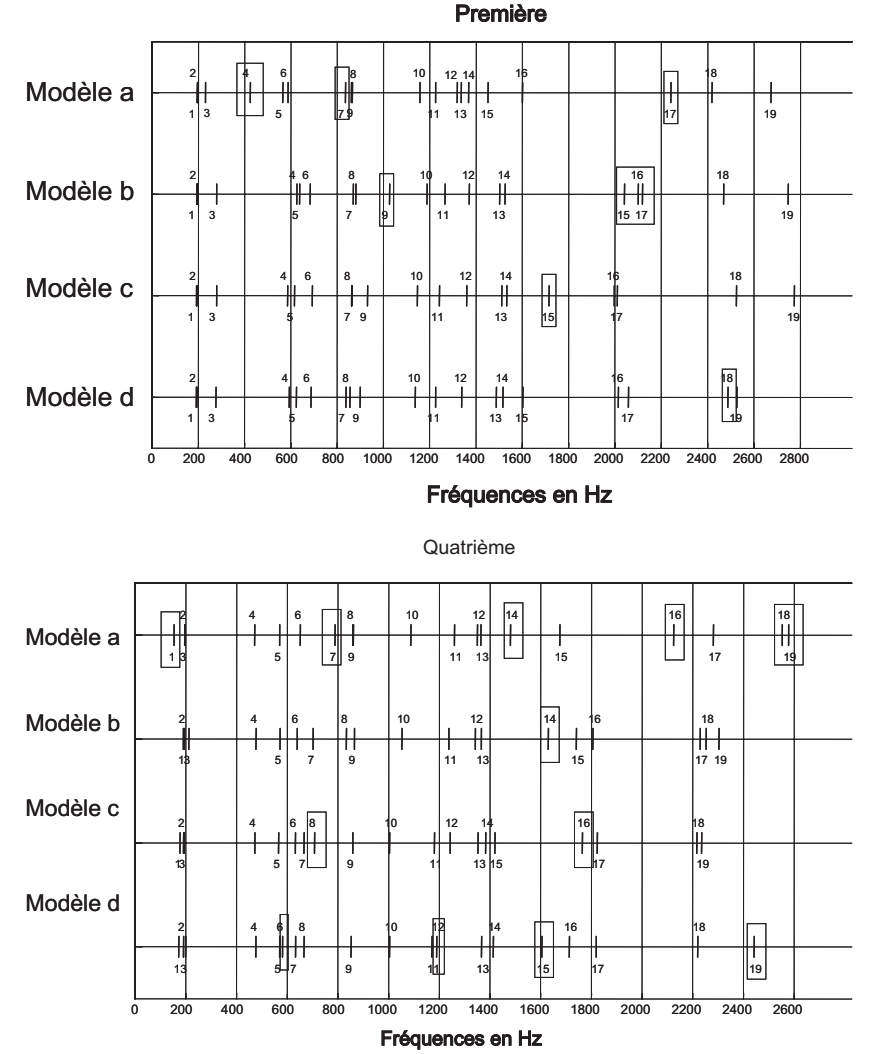

Fig. 5. Comparaison des fréquences propres de chaque modèle en Première et en Quatrième. Les valeurs encadrées indiquent les fréquences spécifiques du modèle.

Tableau 7. Nombre de fréquences propres « caractéristiques ».

\begin{tabular}{cccccc}
\hline & Première & Seconde & Troisième & Quatrième & Cinquième \\
\hline Modèle $\mathrm{a}$ & 3 & 3 & 5 & 6 & 3 \\
Modèle $\mathrm{b}$ & 4 & 1 & 4 & 1 & 2 \\
Modèle c & 1 & 3 & 1 & 2 & 2 \\
Modèle d & 1 & 3 & 1 & 4 & 3 \\
\hline
\end{tabular}

modélisation des roulements. Pour chaque rapport de vitesse il est possible de définir des fréquences spécifiques qui n'apparaissent que dans un seul des 4 modèles de roulements. Le tableau 7 regroupe le nombre de ces fréquences spécifiques pour chaque cas envisagé, ces fréquences sont encadrées sur le diagramme de la figure 5.

Pour l'ensemble des configurations d'engrènement, la répartition des fréquences propres du modèle a se distingue nettement des autres : nombre de fréquences propres spécifiques supérieures aux autres modèles et correspondant à des fréquences basses : pour tous les rapports de vitesses, il y a au moins un mode du modèle a de fréquence inférieure à $800 \mathrm{~Hz}$ qui ne se retrouve pas dans les autres modèles. Dans sa thèse, T.C. Lim [14] compare les résultats modaux obtenus avec des modèles de type a et ceux obtenus avec son propre modèle, comparable au modèle b de cette étude. Il indique que les modèles de type a conduisent à des fréquences propres inférieures à celles de son modèle. Les calculs effectués pour les différentes positions d'engrènement, et donc pour 
des efforts statiques différents, montrent que cela n'est vérifié que dans le cas de la première et de la seconde, c'est-à-dire les cas où les roulements sont les plus chargés, et pour des fréquences propres inférieures à $2000 \mathrm{~Hz}$. Pour les autres positions, les écarts entre les fréquences propres des modèles a et b sont plus complexes. Ces différences entre les conclusions de T.C. Lim et les résultats observés ici peuvent s'expliquer par la présence dans le modèle $b$ de jeux de l'ordre de $8 \times 10^{-3} \mathrm{~mm}$ dans les roulements alors que les valeurs de jeux internes utilisées par T.C. Lim sont beaucoup plus faibles $\left(5 \times 10^{-5} \mathrm{~mm}\right)$. Ces jeux ont, évidemment, un rôle important dans la répartition des charges et donc sur les matrices de rigidité, lorsque les chargements sont faibles.

La prise en compte de la rigidité statique des arbres et des interactions entre les différents paliers à roulement, en particulier les roulements à rouleaux coniques (modèles c et d) conduit à des fréquences propres plus basses que celles obtenues en déterminant les matrices de rigidité des paliers pour des roulements isolés (modèle b). Il semble donc délicat d'utiliser des résultats statiques obtenus sur des roulements isolés pour étudier le comportement dynamique global d'un mécanisme possédant des roulements à contact oblique.

La prise en compte de la rigidité statique du carter (modèle d), n’influence sensiblement que les fréquences propres supérieures à une fréquence « limite » dépendant de l'engrenage considéré et donc de la charge statique appliquée. En première, elle est de $1600 \mathrm{~Hz}$, et de $1200 \mathrm{~Hz}$ en cinquième. En dessous de cette fréquence, les fréquences propres obtenues avec les modèles d et c sont très voisines. Néanmoins, pour des chargements statiques importants (première, seconde, troisième), la prise en compte de la rigidité statique du carter (modèle $\mathrm{d}$ ) conduit à des fréquences propres légèrement plus basses. Cela signifie que dans le cas de chargements statiques importants, la prise en compte de l'environnement statique complet (arbres et carters) diminue la rigidité globale du système.

\section{4 Études des énergies potentielles}

L'examen des diagrammes de répartition en fonction des modes, de l'énergie potentielle totale de flexion, torsion et extension met en évidence les phénomènes de couplages entre les déformées (Figs. 6 et 7 ). Néanmoins, des modes où les effets d'extension sont majoritaires (supérieurs à $90 \%$ ) apparaissent dans tous les modèles lorsque le chargement statique des roulements diminue (troisième, quatrième, cinquième). Lors de l'examen de la répartition des énergies dans les roulements (Fig. 4), il apparaît que ces modes correspondent à des modes très énergétiques pour les roulements. Ce sont donc des modes « pseudo rigides », assimilables à des systèmes masses ressorts, les arbres jouant le rôle de masses indéformables et les roulements jouant celui des ressorts. Les rigidités de ces « ressorts » dépendant du chargement statique appliqué aux roulements, il est donc normal que, pour une bande de fréquences considérée, ces modes « pseudo
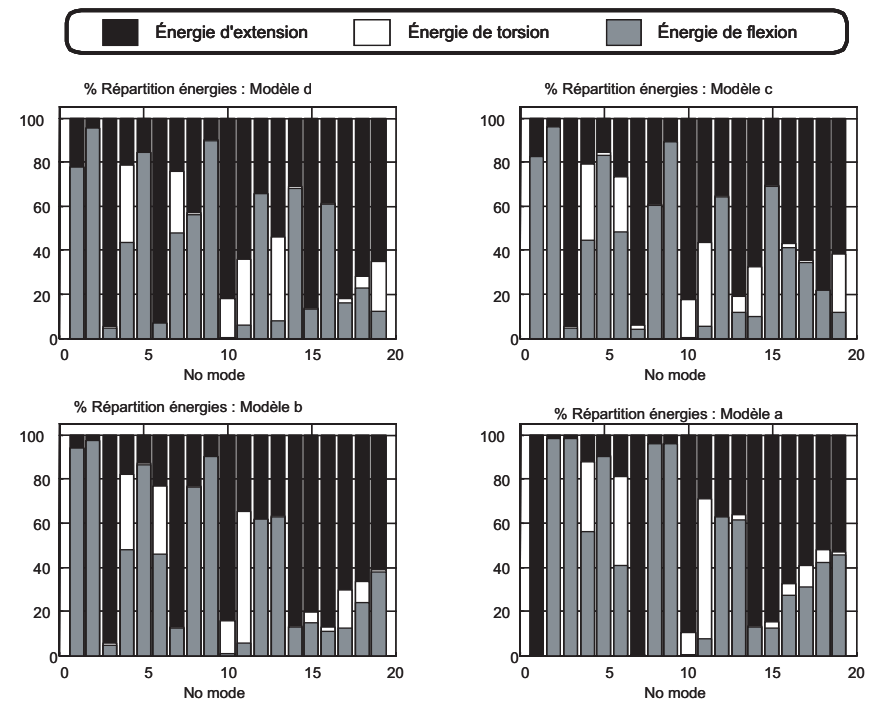

Fig. 6. Participation des énergies de déformations élémentaires en fonction des modes, pour les 4 modèles de roulements en quatrième.

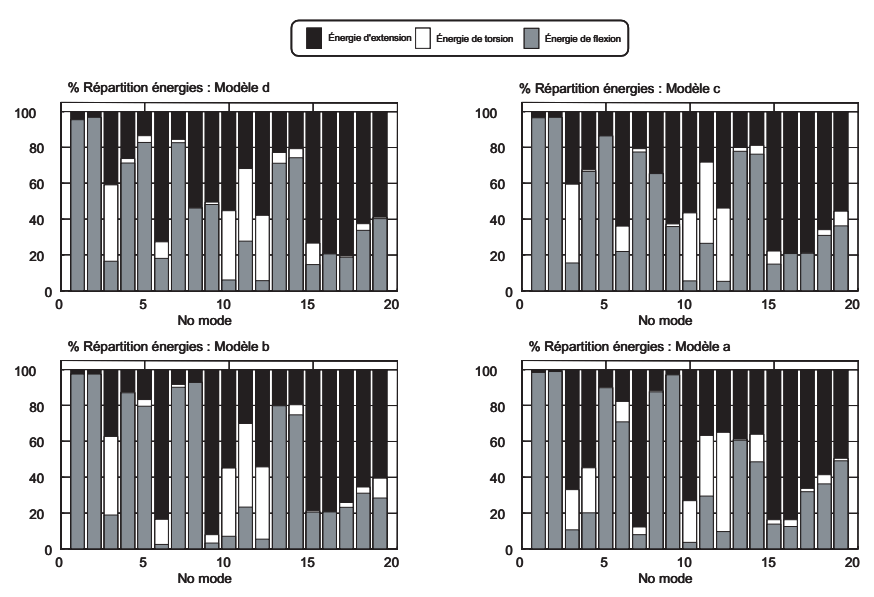

Fig. 7. Participation des énergies de déformations élémentaires en fonction des modes, pour les 4 modèles de roulements en première.

rigides » n'apparaissent que pour certains niveaux de chargement.

Pour l'ensemble des 5 chargements considérés, le diagramme énergétique du modèle a se distingue des autres, en particulier des diagrammes des modèles c et d. En quatrième (Fig. 6), ce modèle possède des modes d'extension pure, sans aucun couplage, (modes 1 et 7 ) qui n'existent dans aucun des autres modèles. Ceci provient des matrices de rigidité associées aux roulements qui, dans le cas du modèle a sont diagonales et n'assurent donc aucun couplage entre les degrés de liberté. Les directions propres de ces matrices sont également les directions propres des arbres. Pour les autres modèles, les matrices de rigidité étant « pleines » leurs directions principales ne sont plus colinéaires à celles des arbres, et les déformations associées doivent nécessairement s'accompagner de flexion ou de torsion, même faible, des arbres. Ces différences 
entre les diagrammes énergétiques, indiquent que les vecteurs propres du modèle a se distinguent des vecteurs des autres modèles par leur forme générale et pas seulement par leur orientation. Les autres modèles, formés par des matrices de rigidité pleines qui assurent ces couplages, possèdent des diagrammes énergétiques se ressemblant davantage.

Les diagrammes énergétiques du modèle b sont plus semblables à ceux des modèles c et d que les diagrammes du modèle a. Néanmoins, des différences apparaissent. Les influences du couplage entre les roulements et de la prise en compte de la déformabilité statique des arbres, se traduisent essentiellement par l'apparition ou la disparition de modes où l'énergie d'extension est majoritaire.

Il existe une bonne ressemblance entre les diagrammes énergétiques des modèles c et d qui doit être mise en relation avec la bonne ressemblance des matrices de MAC correspondantes. Pour les modes appariables avec un MAC supérieur à 0,8 , des différences peuvent apparaître lorsque le MAC est plus proche de 0,8 que de 1 . L'analyse plus détaillée de la répartition énergétique dans les modes appariés montre que les différences sont faibles. Cela confirme le choix de 0,8 comme critère d'appariage. Pour les modes non appariables (MAC inférieur à 0,8) deux cas peuvent apparaître :

- les diagrammes énergétiques sont identiques, les vecteurs propres ont alors la même «forme » mais une orientation différente,

- les diagrammes énergétiques sont différents ce qui traduit un comportement différent : les vecteurs propres ont des formes différentes.

Dans le cas des modèles c et d, l'étude de ces vecteurs non appariés par les matrices de MAC, ne fait pas apparaître la dominante d'un phénomène sur l'autre : $50 \%$ de ces vecteurs pourraient être appariés en forme mais avec une orientation différente, les autres possèdent des diagrammes énergétiques notablement différents. L'influence statique du carter se traduit donc par des orientations différentes de certains vecteurs ou par l'apparition de vecteurs de forme différente bien qu'associés à des fréquences propres voisines.

\section{5 Études des énergies cinétiques}

Les 4 modèles considérés possèdent des matrices de masse identiques, les différences entre les diagrammes des énergies cinétiques proviennent donc uniquement de différences de forme entre les vecteurs propres.

Il est difficile de quantifier globalement l'évolution du partage des énergies d'un modèle à l'autre. En effet, puisque la somme des énergies reste égale à $100 \%$, si un type d'énergie augmente les autres doivent nécessairement diminuer. Néanmoins, l'étude plus spécifique des énergies de torsion est intéressante ici. En effet, les modèles utilisés ne comportent pas d'inerties additionnelles, les déformations de torsion proviennent uniquement des déformations des arbres.

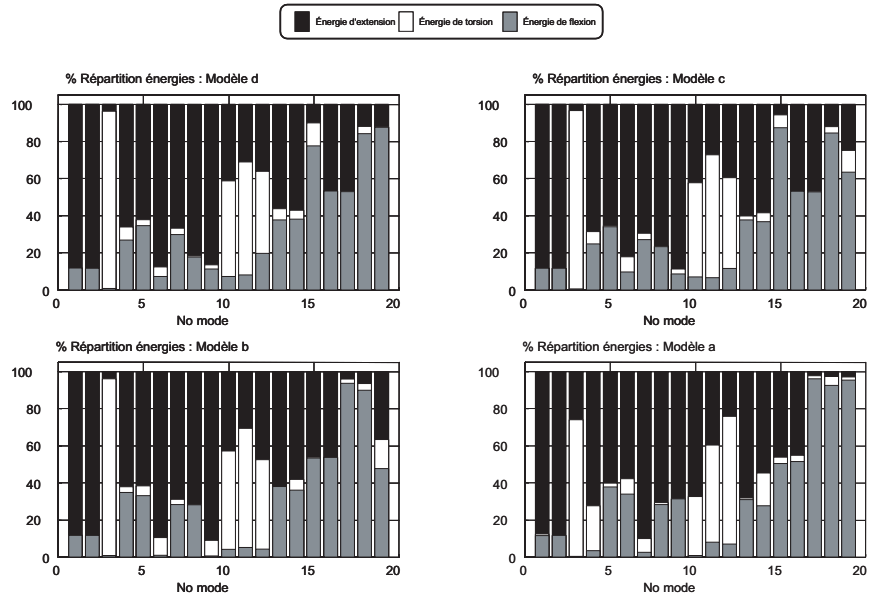

Fig. 8. Participation des énergies cinétiques élémentaires en fonction des modes pour les 4 modèles de roulement en première.
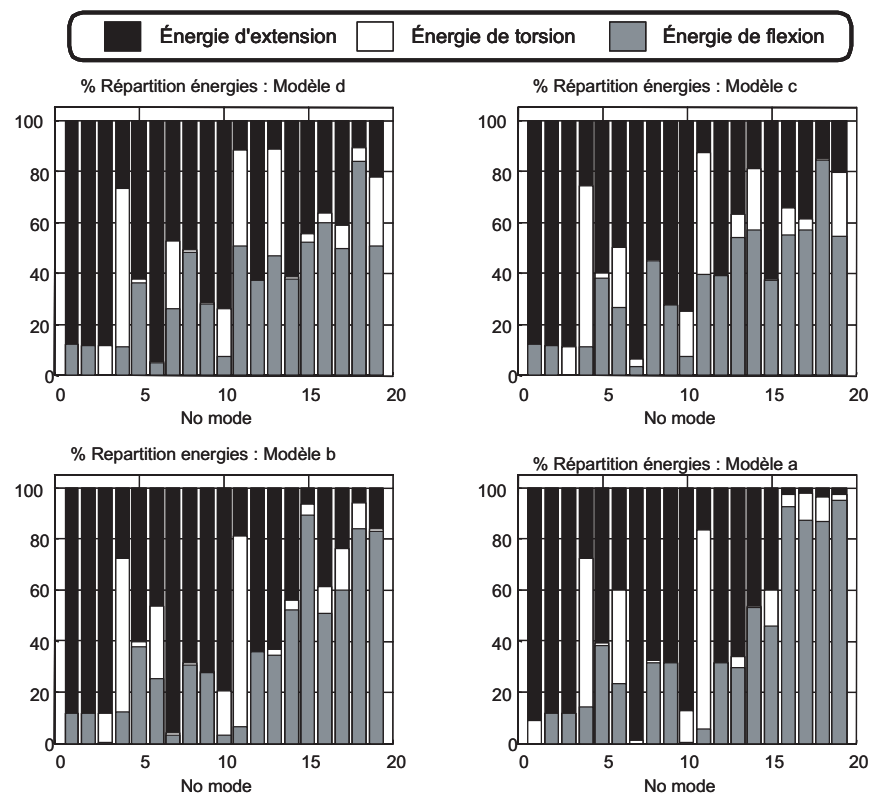

Fig. 9. Participation des énergies cinétiques élémentaires en fonction des modes pour les 4 modèles de roulement en quatrième.

Pour les chargements statiques importants (première, seconde) des différences entre le modèle a et les autres modèles apparaissent dès les basses fréquences (Fig. 8). Pour les modèles $b$, c et $d$, l'énergie cinétique du mode 3 est, pour plus de $60 \%$ de l'énergie de torsion, ce mode ne se retrouve pas dans le modèle a ou bien avec une part d'énergie de torsion beaucoup plus faible (20\%). Pour les chargements statiques plus faibles (troisième, quatrième, cinquième), les différences entre le modèle a et les autres modèles, en particulier les modèles c et d, apparaissent plus nettement pour des fréquences plus élevées mais, là aussi la participation des énergies cinétiques de torsion est modifiée (Fig. 9). Des modes très énergétiques en torsion apparaissent dans le modèle a (mode 11 en 
quatrième) et ne se retrouvent pas dans les modèles c et $\mathrm{d}$ mais éventuellement dans le modèle b.

En première et en seconde et les basses fréquences, le modèle $b$ possède des diagrammes énergétiques très semblables à ceux des modèles c et d. Néanmoins, il apparaît en première des modes, 5 et 9 , où l'énergie d'extension (arbres et masses ponctuelles) est largement majoritaire (90\%) mais où l'énergie de flexion est quasiment nulle. Ce type de mode ne se retrouve pas dans les modèles c et d lorsque le chargement statique est élevé. Pour les autres vitesses, les diagrammes énergétiques du modèle b sont souvent plus proches de ceux du modèle a que de ceux des modèles $\mathrm{c}$ et $\mathrm{d}$. Les modèles $\mathrm{c}$ et $\mathrm{d}$ possèdent des diagrammes énergétiques très voisins. Il apparaît néanmoins une légère augmentation des énergies cinétiques de torsion pour le modèle d surtout sensible pour les fréquences plus élevées.

Pour un même chargement, les différences apparaissant entre les modèles confirment donc que la modélisation des roulements influence fortement la forme des vecteurs propres. La modification de la répartition des énergies cinétiques de torsion prouve que la modélisation statique des roulements influence le comportement en torsion du rotor alors que, paradoxalement, les roulements ne modifient pas les rigidités de rotation axiale du système. Ce phénomène peut s'expliquer par la présence de dentures hélicoïdales et l'introduction de matrices de rigidité «pleines » pour modéliser les roulements. En effet, les dentures hélicö̈dales couplent les déformations de torsion à des déplacements « rigides » axiaux des arbres. Or, à cause des matrices de rigidité « pleines », les directions principales des roulements ne sont plus les directions principales des arbres et ces déplacements axiaux vont obligatoirement être couplés aux autres déformations.

\section{Conclusion}

L'étude bibliographique de la manière dont la rigidité des paliers à roulement est introduite dans les modèles dynamiques des systèmes rotatifs a permis d'identifier trois grands types d'approches : la modélisation par des rigidités scalaires, la modélisation par des matrices pleines obtenues sur des roulements isolés, une modélisation en corps roulants obtenue en prenant en compte la flexibilité de l'environnement. Quatre modélisations ont été retenues et introduites dans un modèle dynamique d'un réducteur multi-étages à engrenages. Des outils spécifiques d'analyse et de comparaison des modes propres : analyse énergétique et matrice de MAC ont été présentés et utilisés pour comparer les modes propres obtenus pour les quatre modèles de roulement.

L'analyse du taux global d'énergie de déformation dans les roulements a confirmé leur rôle prépondérant dans les déformations modales. Ce rôle est d'autant plus important que le chargement statique appliqué est faible. Les modes énergétiques sont nombreux, et relativement bien répartis le long de la bande de fréquences considérée ce qui justifie a posteriori la nécessite d'examiner l'influence de la modélisation retenue pour les roulements.
La comparaison des fréquences propres et des matrices de MAC a permis de montrer que les modèles dynamiques où le comportement des roulements est introduit par des rigidités scalaires équivalentes ou par des matrices obtenues pour des roulements isolés sont insuffisants pour décrire le comportement dynamique du système : les fréquences propres sont différentes, les vecteurs propres ne sont pas colinéaires. Globalement, l'introduction d'un environnement statique flexible arbres ou arbres et carter, par rapport à l'utilisation d'un environnement complètement rigide peut modifier de manière indépendante les fréquences propres et les vecteurs propres ou conduire à l'apparition ou à la disparition de certains modes. Les fréquences propres des modèles dynamiques obtenus en prenant en compte l'environnement statique réel des roulements sont plus faibles que celles obtenues avec les hypothèses d'environnement statique rigide.

L'étude des énergies découplées a montré l'intérêt de modéliser les roulements par des matrices de rigidité complètes plutôt que par des matrices diagonales qui n'assurent pas le couplage des ddl et permettent donc l'apparition de modes de déformation purs. La prise en compte de l'environnement statique des roulements lors de la détermination des matrices de rigidité, va se traduire par l'apparition ou la disparition de modes où l'énergie potentielle d'extension est majoritaire. La prise en compte statique du carter, conduit soit à une modification de l'orientation de certains vecteurs soit à l'apparition de modes de forme très différente. L'étude des énergies cinétiques permet de confirmer que, pour un même chargement statique, la modélisation des roulements va modifier la forme des vecteurs propres. Cette étude a également montré que la modélisation des roulements par des matrices pleines, couplée à l'utilisation d'engrenages hélicoïdaux va influencer le comportement en torsion des arbres.

Une modélisation adéquate des paliers à roulements est donc absolument nécessaire pour représenter correctement le comportement dynamique du système rotatif. La prise en compte de l'environnement réel semble également primordial.

Remerciements. Les auteurs remercient la société PSA qui avait initié ces travaux.

\section{Annexe 1 : Formules de Palmgren}

Les désignations suivantes sont utilisées dans les formules :

$\delta_{\mathrm{r}}$ : déplacement radial en $\mathrm{mm}$

$\delta_{\mathrm{a}}$ : déplacement axial en $\mathrm{mm}$

$F_{\mathrm{r}}$ : charge radiale en daN

$F_{\mathrm{a}}$ : charge axiale en da $\mathrm{N}$

$D_{w}$ : diamètre des éléments roulants en $\mathrm{mm}$

$D_{\mathrm{r}}$ : diamètre nominal du roulement en $\mathrm{mm}$

$l_{a}$ : longueur effective des rouleaux en $\mathrm{mm}$

$Z$ : nombre d'éléments roulants

$i$ : nombre de rangées d'éléments roulants. 
Tableau A.1. Formules de Palmgren. $\alpha$ : angle de contact, $Q$ : charge maximale sur les éléments roulants en daN. Les déformations élastiques $\delta_{\mathrm{r}}$ et $\delta_{\mathrm{a}}$ dans le roulement s'expriment comme suit.

\begin{tabular}{lcc}
\hline & \multicolumn{2}{c}{ Cas de charge } \\
& radiale $: \delta_{\mathrm{a}}=0$ & axiale $\delta_{\mathrm{r}}=0$ \\
\hline Charge sur éléments roulants & $Q=\frac{5 F_{\mathrm{r}}}{i \cdot z \cdot \cos \alpha}$ & $Q=\frac{5 F_{\mathrm{a}}}{z \cdot \sin \alpha}$ \\
\hline Type de roulement & $\delta_{\mathrm{r}}=\frac{0,0032}{\cos \alpha} \sqrt[3]{\frac{Q^{2}}{D_{w}}}$ & - \\
Rotule sur billes & $\delta_{\mathrm{r}}=0,002 \sqrt[3]{\frac{Q^{2}}{D_{w}}}$ & - \\
Rigides à billes & $\delta_{\mathrm{r}}=\frac{0,002}{\cos \alpha} \sqrt[3]{\frac{Q^{2}}{D_{w}}}$ & $\delta_{\mathrm{a}}=\frac{0,002}{\sin \alpha} \sqrt[3]{\frac{Q^{2}}{D_{w}}}$ \\
$\begin{array}{l}\text { Billes à contact oblique } \\
\begin{array}{l}\text { Rouleaux à contact linéaire sur les } \\
\text { deux pistes }\end{array}\end{array}$ & $\delta_{\mathrm{r}}=\frac{0,0006}{\cos \alpha} \frac{Q^{0,9}}{l_{a}^{0,8}}$ & $\delta_{\mathrm{a}}=\frac{0,0006}{\sin \alpha} \frac{Q^{0,9}}{l_{a}^{0,8}}$ \\
$\begin{array}{l}\text { Rouleaux à contact linéaire sur une } \\
\text { piste, ponctuel sur l'autre }\end{array}$ & $\delta_{\mathrm{r}}=\frac{0,0012}{\cos \alpha} \frac{Q^{3 / 4}}{l_{a}^{1 / 2}}$ & $\delta_{\mathrm{a}}=\frac{0,0012}{\sin \alpha} \frac{Q^{3 / 4}}{l_{a}^{1 / 2}}$ \\
Butées à billes & - & $\delta_{\mathrm{a}}=\frac{0,024}{\sin \alpha} \sqrt[3]{\frac{Q^{2}}{D_{w}}}$ \\
\hline
\end{tabular}

Tableau A.2. Formules de Krämer.

\begin{tabular}{|c|c|c|c|}
\hline \multicolumn{4}{|c|}{ Raideur radiale $: k_{\mathrm{r}}=\frac{n \cdot F_{\mathrm{r}}}{x}$} \\
\hline & $n$ & $x$ & $k_{\mathrm{r}}$ \\
\hline $\begin{array}{l}\text { Roulement à billes } \\
\text { Roulement à rouleaux }\end{array}$ & $\begin{array}{l}n=\frac{3}{2} \\
n=\frac{1}{0,9}\end{array}$ & $\begin{array}{l}x=1,2^{\prime} \times 10^{-7 \prime} d^{-\frac{1}{3} \prime} Z^{-\frac{2}{3} \prime} F^{\frac{2}{3}} \\
x=1,11^{\prime} \times 10^{-9 \prime} l^{-0,8 \prime} Z^{-0,9 \prime} F^{0,9}\end{array}$ & $\begin{array}{l}k=1,25^{\prime} \times 10^{7 \prime} d^{\frac{1}{3} \prime} Z^{\frac{2}{3} \prime} F^{\frac{1}{3}} \\
k=1,00^{\prime} \times 10^{9 \prime} l^{0,8 \prime} Z^{0,9 \prime} F^{0,1}\end{array}$ \\
\hline \multicolumn{4}{|c|}{ Raideur axiale $: k_{\mathrm{a}}=r \cdot K_{\mathrm{r}}$} \\
\hline $\begin{array}{l}\text { Roulement à billes } \\
\text { Roulement à rouleaux }\end{array}$ & \multicolumn{3}{|c|}{$\begin{array}{l}r=-0,0028 Z^{2}+0,0987 Z-0,12 \\
r=-0,0028 Z^{2}+0,1012 Z-0,17\end{array}$} \\
\hline
\end{tabular}

\section{Annexe 2 : Formules de Krämer}

Les désignations suivantes sont utilisées dans les formules ci-dessus :

$$
\begin{aligned}
& x: \text { déplacement }(\mathrm{m}) \\
& k: \text { raideur }\left(\mathrm{N} \cdot \mathrm{m}^{-1}\right) \\
& d: \text { diamètre billes }(\mathrm{m}) \\
& l: \text { longueur rouleaux }(\mathrm{m}) \\
& Z: \text { nombre d'éléments roulants } \\
& F: \text { charge }(\mathrm{N}) .
\end{aligned}
$$

\section{Références}

[1] R. Maliha, C.U. Dogruer, H.N. Özgüven, Non-linear dynamic modelling of gear-shaft-disk-bearing systems using finite elements and describing functions, ASME J. Mechanical Design 126 (2004) 534-541

[2] E. Tanaka, N. Tanaka, K. Ohno, Vibration analysis of a multi-stage gear system including drive mechanism elements, JSME 44 (2001) 307-314

[3] Y. Cheng, T.C. Lim, Dynamics of Hypoid gear transmission with nonlinear time-varying mesh characteristics, ASME J. mechanical design 125 (2003) 373-382
[4] A. Al-shyyab, A. Kahraman, Non-linear dynamic analysis of a multi-mesh gear train using multi-term harmonic balance method: sub-harmonic motions, J. Sound and Vibration 284 (2005) 417-451

[5] M. Li, L. Yu, Analysis of the coupled lateral torsional vibration of a rotor-bearing system with a misaligned gear coupling, J. Sound and Vibration 243 (2001) 283-300

[6] M. Li, H.Y. Hu, P.L. Jiang, L. Yu, Coupled axial-lateraltorsional dynamics of a rotor-bearing system geared by spur bevel gears, J. Sound and Vibration 254 (2002) 427-446

[7] M. Li, H.Y. Hu, Dynamic analysis of a spiral bevelgeared-rotor-bearing system, J. Sound and Vibration 259 (2003) 605-624

[8] M. Kubur, A. Kahraman, D.M. Zini, K. Kienzle Dynamic Analysis of a multi-shaft helical gear transmission by finite elements: model and experiment, ASME J. Vibration and Acoustics 126 (2004) 398-406

[9] E. Tanaka, H. Houjoh, D Mutoh, H. Motoshiromitzu, K. Ohno, N. Tanaka, Vibration and sound-radiation analysis for designing a low noise gearbox with multi-stage helical gear system, JSME 46 (2003) 1178-1185

[10] B.L. Choi, J.M. Park, An improved rotor model with equivalent dynamic effects of the support structure, J. Sound and Vibration 244 (2001) 569-581 
[11] Y. Kang, Y.P. Chang, J.W. Tsai, L.H. Mu, Y.F. Chang, An investigation in stiffness effects on dynamics of rotorbearing-foundation systems, J. Sound and Vibration 231 (2000) 343-374

[12] Y. Cheng, T.C. Lim, Dynamics of Hypoid gear transmission with nonlinear time-varying mesh characteristics, J. Sound and Vibration 240 (2001) 519-543

[13] E. Rigaud, F. Mayeux, N. Driot, J. Perret-Liaudet, B. Mevel, Variabilité des régimes critiques d'une boîte de vitesses : influence des précharges des roulements, Mécanique \& Industries 4 (2003) 107-112

[14] T.C. Lim, Vibration transmission through rolling element bearing in geared rotor system, Ph.D. Thesis Ohio State University, 1989

[15] P. Dietl, J. Wensing, G.C. van Nijen, Rolling bearing damping for dynamic analysis of multi-body systems - Experimental and theoretical results, J. Multi-body Dynamic 214 (2000) 33-43

[16] D. Remond, P. Velex, J. Sabot, Comportement dynamique et acoustique des transmissions par engrenages, Synthèse bibliographique, Senlis : Publication CETIM, 1993

[17] K. Umezawa, H. Houjoh, N. Ichikawa, S. Matsumura, Simulation of rotational vibration of a helical gear pair transmitting light loads, in Proceedings of the 3rd JSME International Conference on Motion and Power transmissions MPT'91, Hiroshima, Japan, 1991, pp. 85-91

[18] K. Umezawa, H. Houjoh, N. Ichikawa, S. Matsumura, The influence of flank deviations on the vibration of a helical gear pair transmitting light loads, in Proceedings of the 6th ASME International Conference Power Transmissions and gearing, Phoenix 2, 1992, pp. 681-688

[19] S.T. Choi, S.Y. Mau, Dynamic analysis of geared rotorbearing systems by the transfer matrix method, ASME J. Mechanical Design 123 (2001) 562-568

[20] F. Peeters, R. Pintelon, J. Schoukens Y. Rolain, E.S. Gutierrez, P. Guillaume, Identification of rotor bearing systems in the frequency domain, Part 1: Estimation of frequency response functions, Mechanical Systems and Signal Processing 15 (2001) 759-773

[21] F. Peeters, R. Pintelon, J. Schoukens, Y. Rolain, Identification of rotor bearing systems in the frequency domain, Part II: Estimation of modal parameters Mechanical Systems and Signal Processing 15 (2001) $775-788$

[22] H.N. Ozguven, On the critical speed of continuous shaftdisk system, ASME J. Vibration, Acoustics, Stress, and Reliability in Design 106 (1984) 59-61

[23] J.S. Rao, Rotor dynamics, John Wiley, New-York, 1983

[24] B. Moon, B.S. Kang Non Linear Vibration analysis of mechanical system (analysis with consideration of nonlinear sensitivity), JSME 44 (2001) 12-19.

[25] A. Palmgren, Les roulements descriptions, théorie, applications, SKF 1967, 120 p.

[26] E. Krämer, Dynamics of rotors and foundations, Springer Verlag, 1993, 383 p.

[27] E.R. Marsh, D.S. Yantek, Experimental measurement of precision bearing dynamic stiffness, J. Sound and Vibration 202 (1997) 55-66

[28] T.C. Lim, R. Singh Vibration transmission through rolling element bearings. Part I: Bearing stiffness formulation, Part II: System studies, Part III: Gear rotor system studies, J. Sound and Vibrations 139 (1990)
[29] J. Perret-Liaudet, Étude des mécanismes de transfert entre l'erreur de transmission et la réponse dynamique des boîtes de vitesses automobiles, Thèse École Centrale de Lyon, 1992

[30] A. Bourdon, Modélisation dynamique globale des boîtes de vitesses automobile, Thèse : Institut National des Sciences Appliquées de Lyon 1997. Disponible en ligne arXiv:http://docinsa.insa-lyon.fr/these/ pont.php ?id=bourdon

[31] C. Bard, Modélisation du comportement dynamique des transmissions de puissance par engrenages, Thèse : Institut National des Sciences Appliquées de Lyon, 1995

[32] M.D. Rajab, Of the transmissibility through rollingelement bearing under radial and moment loads Modelling, These, Ohio State University, 1982

[33] W.B. Young. Dynamic modelling and experimental measurements of a gear shaft and housing system, These, Ohio State University, 1988

[34] Y. Kang, Y.P. Chang, J.W. Tsai, L.H. Mu, Y.F. Chang, An investigation in stiffness effects on dynamics of rotorbearing-foundation systems, J. Sound and Vibration 231 (2000) 343-374

[35] J.M. de Mul, J.M. Vree, D.A. Mass, Equilibrum and associated load distribution in ball and roller bearing in five degrees of freedom while neglecting friction, Part I and II, ASME J. Tribology 111 (1989) 142-155

[36] A. Bourdon, J.F. Rigal, D. Play, Static rolling bearing models in C.A.D. environment for the study of complex mechanisms, Part I - Rolling bearing model, Part II Complete assembly model ASME J. Tribology 121 (1999) 205-223

[37] S. Andreason, Load distribution in a taper roller bearing arrangement considering misalignement, Tribology 6 (1973) 84-92

[38] T.A. Harris, Rolling bearing analysis, 2nd edition, New York: John Wiley \& Sons, 1984

[39] T.A. Harris, The effect of misalignement on the fatigue life of a cylindrical roller bearing having crowned roller members, ASME J. Lubrification Technology (1969) 294300

[40] J.Y. Liu, The effect of misalignement on the life of high speed cylindrical roller bearings, ASME J. Lubrification Technology (January 1970) 129-137

[41] J.Y Liu, Y.P. Chiu, Analysis of a thin elastic ring under arbitrary loading, ASME J. Engineering for Industry (August 1974) 870-876

[42] X. Hernot, M. Sartor, J. Guillot, Calculation of the stiffness matrix of angular contact ball bearings by using the analytical approach, ASME J. Mechanical Design 122 (2000) 83-90

[43] A.B. Jones, J.M. Mc Grew, Rotor bearings dynamic technology design Guide: Part II, Ball bearings, Wright Patterson, Ohio, USA: Air Force Aero Propulsion Laboratory, AFAPL-TR-78-6 1978

[44] A.B. Jones, J.M. Mc Grew, Rotor bearings dynamic technology design Guide: part III Tapered roller bearings, Wright Patterson, Ohio, USA: Air Force Aero Propulsion Laboratory, AFAPL-TR-78-6, 1979, 80 p.

[45] A.B. Jones, J.M. Mc Grew, Rotor bearings dynamic technology design Guide: part IV, cylindrical roller bearing, Wright Patterson, Ohio, USA: Air Force Aero Propulsion Laboratory, AFAPL-TR-78-6, 1979, 61 p. 
[46] L. Houpert, Prediction of bearing, gear and housing performances. Rolling Bearing Practice Today seminar, I. Mech. E. London, 1995

[47] A. Bourdon, K. Yakhou, L. Chaloyrad, D. Play, Influence of the mechanical architecture and coupling effects on the vibratory behavior of complex gear transmission, in Proceedings of DETC99: 1999 ASME Design Engineering Technical Conferences, September 12-15, 1999, Las Vegas, Nevada

[48] K. Yakhou, A. Bourdon, D. Play Prediction of Automotive Gearboxes Dynamic Behavior, Part A: Experimental Validation of a Global Numerical Model, in proceeding of DETC2000/PTG-14428, 8th International ASME Power Transmission and Gearing Conference, September 10-13, 2000, Baltimore

[49] A. Bourdon, K. Yakhou, D. Play, Prediction of Automotive Gearboxes Dynamic Behavior, Part B : Numerical Simulations for the Determination of New
Mechanical Design Trends, in proceeding of DETC2000/PTG-14427, 8th International ASME Power Transmission and Gearing Conference, September 10-13, 2000, Baltimore

[50] K. Yakhou, Analyse des matrices de rigidité des paliers à roulements, mémoire de DEA, INSA de Lyon, Laboratoire CASM, 1995

[51] A. Girad, Modal effective mass models in structural dynamics, in proceeding of: Xth International Modal Analysis Conference, San Diego USA (IMAC), 1992

[52] A. Bourdon, J.F. Rigal, D. Play, Numerical modal result processings using CAD for analysing global vibrating behavior of gearboxes, in Proceedings of the International Congress of Gear Transmissions'95, IftomEurotrans, Sophia, 2, 1995, pp. 104-110

[53] B. Mevel, Comportement dynamique des roulements a billes, Thèse : Institut National des Sciences Appliquées de Lyon, 1992 